\title{
PRICING AND HEDGING CONTINGENT CLAIMS WITH REGIME SWITCHING RISK*
}

\author{
ROBERT J. ELLIOTT ${ }^{\dagger}$ AND TAK KUEN SIU ${ }^{\ddagger}$
}

\begin{abstract}
We study the pricing and hedging of contingent claims in a Markov regime-switching market with a money market account, a zero-coupon bond, and an ordinary share. General contingent claims with payoffs depending on both the share price and the state of a Markov chain describing regime switching are considered. A general pricing kernel defined by the product of two density processes is used to explicitly take into account regime switching risk. Under some differentiability and boundedness conditions, a martingale representation result is established and the integrands in the representation are explicitly identified with respect to the general pricing kernel. We then determine a pricing kernel and a hedging strategy by minimizing the residual risk due to incomplete hedging. Our analysis is also extended to Asian-style and American-style general contingent claims.
\end{abstract}

Key words. Contingent claims, regime switching risk, valuation, hedging, product density processes, martingale representation, stochastic flows, zero-coupon bonds, residual risk, Asian options, American options.

AMS subject classifications. $60 \mathrm{H} 30$.

\section{Introduction}

Recently, regime-switching models have attracted attention from both academia and practitioners. The basic structure of the models is that the set of model parameters in force at a particular time depends on the state of a driving process, say a Markov chain. When there is a switch in the state of the driving process, the set of model parameters changes to another set. The states of the driving process are usually interpreted as different states of an economy. Consequently, regime switching models can be used to describe the impact of structural changes in (macro)-economic conditions on the model dynamics. Their practical importance are exemplified by some discussions about the recent global financial crisis, starting from mortgages and housing bubbles in the United States, where the importance of incorporating structural changes in economic conditions in economic and financial models is highlighted. The history of regime switching models may be traced to the early works of Quandt [26] and Goldfeld and Quandt [18], where a regime-switching version of a regression model was used to fit nonlinear economic data. The idea of regime switching also appeared in works of Tong $[28,29,30]$ on nonlinear time series analysis, where the subject was still in its infancy. He introduced a class of threshold time series models, where regime switching was governed by a threshold principle. Hamilton [20] pioneered and popularized econometric applications of an important class of regime switching models, namely, the class of discrete-time, Markov-switching, autoregressive models. Regime switching models have some empirical advantages. They describe many important "stylised" features of economic and financial time series, such as the asymmetry and heavy-tailedness of distributions for assets' returns, time-varying conditional volatility, volatility clustering, and regime switching.

Incorporating the risk attributed to structural changes in (macro)-economic conditions in the pricing and hedging of contingent claims is an important issue. Failure

\footnotetext{
*Received: June 4, 2010; accepted: September 18, 2010. Communicated by David Cai.

${ }^{\dagger}$ School of Mathematical Sciences, University of Adelaide, SA 5005 Australia; Haskayne School of Business, University of Calgary, Calgary, Alberta, Canada (relliott@ucalgary.ca).

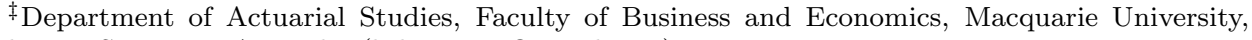
Sydney, NSW 2109, Australia (ktksiu2005@gmail.com).
} 
to incorporate regime-switching risk may lead to the underestimation of derivatives' prices and inappropriate methods to hedge and manage risk. This may then lead to pervasive risk-taking activities which produce factors causing financial crises. The pricing and hedging of options in a regime-switching environment has been studied by several authors. Some of them include Naik [24], Guo [19], Buffington and Elliott [5], Elliott et al. [13], Elliott et al. [14, 15], Boyle and Draviam [4], Siu [27], Elliott and Siu [16], and others. A market model which includes a regime-switching component describing changes in economic environments is generally incomplete in the sense that not all derivative securities can be perfectly hedged. The pricing and hedging of contingent claims in this market are then complicated issues. Guo [19] introduced an approach based on the completion of the market by a set of fictitious securities. Elliott et al. [13] introduced an approach based on the Esscher transform for option valuation in a market including a regime-switching component. However, both approaches seem not to provide an appropriate method to price explicitly the risk due to structural changes.

Besides pricing, the hedging of contingent claims in a regime-switching environment has also been considered in the literature. Elliott et al. [14] considered the pricing and hedging of a European-style contingent claim in a regime-switching environment. In Elliott et al. [13], they established a martingale representation for the price of the claim evaluated using a pricing kernel selected by a version of a regimeswitching Esscher transform. The integrands in the martingale representation were identified using the concept of stochastic flows. The martingale representation result in Elliott et al. [14] can be considered an extension of that in Elliott and Föllmer [11] and Colwell et al. [6] in a regime-switching environment. However, the hedging strategy developed in Elliott et al. [14] was different from those in Elliott and Föllmer [11] and Colwell et al. [6]. In Elliott et al. [14], a zero-coupon bond was introduced and the hedging strategy was developed by minimizing the residual risk due to incomplete hedging. Elliott and Siu [16] developed a pricing and hedging method for a regime-switching model, where the regime switching process causes jumps in the share price and structural changes in the model parameters at the same time. This model is a generalization of one considered in Naik [24]. The approach used in Elliott and Siu [16] is related to that in Colwell and Elliott [7], where the pricing and hedging of a contingent claim in a jump-diffusion market was considered. The hedging strategy in Elliott and Siu [16] was developed based on the local-minimization of the hedging cost adopted in Colwell and Elliott [7]. It was different from the hedging approach based on the zero-coupon bond in Elliott et al. [14].

In this paper we study the pricing and hedging of contingent claims in a Markov regime-switching model with a money market account, a zero-coupon bond, and an ordinary share. In such model, the market interest rate of the money market account, the appreciation rate and volatility of the share are modulated by a continuous-time, finite-state, Markov chain. The states of the chain may represent different macroeconomic conditions, market conditions over the business cycle, policy regimes and credit ratings of firms or countries. There are two sources of risk in the Markov, regime-switching, market. One source of risk is due to fluctuations of market prices and is referred to as financial risk. Another source of risk is due to transitions of economic conditions and is referred to as economic risk. To price and hedge a contingent claim in the Markov, regime-switching market, it is important to take into account the two sources of risk. Here we introduce a general pricing kernel defined by the product of two density processes: one for a measure change for a diffusion process 
and another one for a measure change for a Markov chain. This general pricing kernel explicitly takes into account both the financial and economic risks. General contingent claims with payoffs depending on both the share price and the state of a Markov chain are considered. An example of such contingent claims is an option whose payoff depends on both the prices of an ordinary share issued by a corporation and its corporate credit ratings issued by a specified rating agency. Under some differentiability and boundedness conditions, a martingale representation result is established and the integrands in the representation are explicitly identified with respect to the general pricing kernel. We then determine a pricing kernel and a hedging strategy by minimizing the residual risk due to incomplete hedging, where the residual risk is measured by the variance of hedging error. Our analysis is also extended to Asian-style and American-style general contingent claims.

This paper is an extension of the results in Elliott et al. [14]. The regimeswitching Esscher transform used in Elliott et al. [14] could not price explicitly both the financial and economic risks in establishing the pricing and hedging strategy of a contingent claim. This may be seen from the fact that the rate matrix of the underlying Markov chain remains unchanged under a measure change by the regime-switching Esscher transform. Here, instead of using the regime-switching Esscher transform, the pricing kernel based on the product of two density processes is used. Only Europeanstyle contingent claims were considered in the existing literature including Elliott and Föllmer [11], Colwell et al. [6], Colwell and Elliott [7], Elliott et al. [14] and Elliott and Siu [16]. Here we extend the pricing and hedging results to Asian-style and American-style contingent claims. We also consider a general form of contingent claims whose payoffs depend on the state of the regime switching process.

The rest of this paper is organized as follows. Section 2 presents the model dynamics of the Markov, regime-switching market. In Section 3, we introduce the general pricing kernel. In Section 4, we derive the martingale representation result and identify the integrands in the representation. Section 5 discusses the pricing and hedging of a general contingent claim. Extensions of the results to the Asianstyle and American-style general claims are developed in Section 6. The final section summarizes the paper.

\section{The Markov, regime-switching, market}

We consider a simple, continuous-time, financial market with two primitive securities, namely, a money market account $B$ and an ordinary share $S$. These securities are supposed to be traded continuously over time through a finite time horizon $\mathcal{T}:=[0, T]$, where $T \in(0, \infty)$. To model uncertainty, we fix a complete probability space $(\Omega, \mathcal{F}, \mathcal{P})$, where $\mathcal{P}$ is a real-world probability measure. We suppose that the probability space is rich enough to model both the financial and economic risks.

To model changing macro-economic conditions, we consider a continuous-time, finite-state, observable Markov chain $\mathbf{X}:=\{\mathbf{X}(t) \mid t \in \mathcal{T}\}$ on $(\Omega, \mathcal{F}, P)$ with state space $\mathcal{S}:=\left\{\mathbf{s}_{1}, \mathbf{s}_{2} \ldots, \mathbf{s}_{N}\right\} \in \Re^{N}$. Therefore, an $N$-regime economy is considered. Following the convention in Elliott et al. [10], we identify, without loss of generality, the state space of the chain $\mathbf{X}$ with a finite set of standard unit vectors $\mathcal{E}:=\left\{\mathbf{e}_{1}, \mathbf{e}_{2}, \ldots, \mathbf{e}_{N}\right\} \in \Re^{N}$, where the $j^{\text {th }}$ component of $\mathbf{e}_{i}$ is the Kronecker delta $\delta_{i j}$, for each $i, j=1,2, \ldots, N$. The space $\mathcal{E}$ is called a canonical state space of the Markov chain.

To specify the probability law of the chain $\mathbf{X}$ under $P$, we define a family of rate matrices $\mathbf{A}(t):=\left[a_{i j}(t)\right]_{i, j=1,2, \cdots, N}, t \in \mathcal{T}$, of the chain $\mathbf{X}$. These rate matrices are also called generators, transition intensity matrices, or $Q$-matrices. For each $t \in \mathcal{T}$ and each $i, j=1,2, \cdots, N$ with $i \neq j, a_{j i}(t)$ is the instantaneous transition intensity of the 
chain from state $\mathbf{e}_{i}$ to state $\mathbf{e}_{j}$ at time $t$. The instantaneous transition intensities $a_{j i}(t), t \in \mathcal{T}$ and $i, j=1,2, \cdots, N$, must satisfy the following properties:

1. $a_{j i}(t) \geq 0$;

2. $\sum_{j=1}^{N} a_{j i}(t)=0$, so $a_{i i}(t) \leq 0$.

Here we assume that $a_{i j}(t)>0$, for $i \neq j$, so $a_{i i}(t)<0$. This assumption excludes some degenerate cases.

Let $F^{\mathbf{X}}:=\left\{\mathcal{F}^{\mathbf{X}}(t) \mid t \in \mathcal{T}\right\}$, which represents the $P$-completed natural filtration generated by $\mathbf{X}$. With the canonical state space of the chain $\mathbf{X}$, Elliott et al. [10] gave the following semimartingale dynamics for the chain $\mathbf{X}$ under $P$ :

$$
\mathbf{X}(t)=\mathbf{X}(0)+\int_{0}^{t} \mathbf{A}(u) \mathbf{X}(u-) d u+\mathbf{M}(t) .
$$

Here $\mathbf{M}:=\{\mathbf{M}(t) \quad \mid \quad t \in \mathcal{T}\} \quad$ is an $\Re^{N}$-valued $\left(F^{\mathbf{X}}, P\right)$-martingale. Since $\int_{0}^{t} \mathbf{A}(u) \mathbf{X}(u-) d u, t \in \mathcal{T}$, is a predictable process of bounded variation, $\mathbf{X}$ is a special semimartingale, and the above semimartingale decomposition is unique. The semimartingale dynamics of the chain $\mathbf{X}$ will be used in the specification of a pricing kernel in Section 3.

We now present the price dynamics of the money market account $B$ and the share $S$. For each $t \in \mathcal{T}$, let $r(t)$ be the instantaneous interest rate of the money market account $B$. We suppose that $r(t)$ is determined by the chain $\mathbf{X}$ as

$$
r(t):=\langle\mathbf{r}, \mathbf{X}(t)\rangle, \quad t \in \mathcal{T} .
$$

Here $\mathbf{r}:=\left(r_{1}, r_{2}, \cdots, r_{N}\right)^{\prime} \in \Re^{N}$ with $r_{i}>0$, for each $i=1,2, \cdots, N, \mathbf{y}^{\prime}$ is the transpose of a matrix, or a vector $\mathbf{y} ; r_{i}$ is instantaneous interest rate of the money market account when the economy is in the $i^{\text {th }}$ state; and the scalar product $\langle\cdot, \cdot\rangle$ in $\Re^{N}$ selects the component of $\mathbf{r}$ that is in force at a particular time based on the state of the economy at that time.

The balance of the money market account $B:=\{B(t) \mid t \in \mathcal{T}\}$ with an unit initial investment evolves over time as

$$
B(t)=\exp \left(\int_{0}^{t} r(u) d u\right), \quad t \in \mathcal{T}, \quad B(0)=1 .
$$

Let $W:=\{W(t) \mid t \in \mathcal{T}\}$ be a standard Brownian motion on $(\Omega, \mathcal{F}, P)$ with respect to the $\mathcal{P}$-completion of its own natural filtration, denoted as $F^{W}:=\left\{\mathcal{F}^{W}(t) \mid t \in \mathcal{T}\right\}$. Here $W$ represents random shocks in financial prices and is the source of financial risk, whereas $\mathbf{X}$ describes transitions of macro-economic conditions and is the source of economic risk. In practice, the financial and economic risks may be correlated. However, to simplify our discussion, we suppose that $W$ and $\mathbf{X}$ are stochastically independent under $P$.

Let $\mu(\cdot, \cdot, \cdot): \mathcal{T} \times \Re^{+} \times \mathcal{E} \rightarrow \Re$ and $\sigma(\cdot, \cdot, \cdot): \mathcal{T} \times \Re^{+} \times \mathcal{E} \rightarrow \Re^{+}$be measurable functions such that for each $t \in \mathcal{T}$ and $i=1,2, \cdots, N, \mu\left(t, S, \mathbf{e}_{i}\right)$ and $\sigma\left(t, S, \mathbf{e}_{i}\right)$ are three times differentiable in $S$ and which, together with their derivatives, are bounded. Here $\Re^{+}$ represents the positive real line. For each $i=1,2, \cdots, N, \mu\left(t, S, \mathbf{e}_{i}\right)$ and $\sigma\left(t, S, \mathbf{e}_{i}\right)$ are the appreciation rate and the volatility of the share, respectively, when the economy is in the $i^{t h}$ state. Then,

$$
\begin{aligned}
& \mu(t, S, \mathbf{X}(t)):=\langle\boldsymbol{\mu}(t, S), \mathbf{X}(t)\rangle \\
& \sigma(t, S, \mathbf{X}(t)):=\langle\boldsymbol{\sigma}(t, S), \mathbf{X}(t)\rangle
\end{aligned}
$$


where

$$
\begin{aligned}
& \boldsymbol{\mu}(t, S):=\left(\mu\left(t, S, \mathbf{e}_{1}\right), \mu\left(t, S, \mathbf{e}_{2}\right), \cdots, \mu\left(t, S, \mathbf{e}_{N}\right)\right)^{\prime} \in \Re^{N}, \\
& \boldsymbol{\sigma}(t, S):=\left(\sigma\left(t, S, \mathbf{e}_{1}\right), \sigma\left(t, S, \mathbf{e}_{2}\right), \cdots, \sigma\left(t, S, \mathbf{e}_{N}\right)\right)^{\prime} \in \Re^{N} .
\end{aligned}
$$

We suppose that, under $P$, the share price process $S:=\{S(t) \mid t \in \mathcal{T}\}$ evolves over time according to the following Markov, regime-switching, geometric Brownian motion $(\mathrm{GBM})$ :

$$
\begin{aligned}
d S(t) & =\mu(t, S(t), \mathbf{X}(t)) S(t) d t+\sigma(t, S(t), \mathbf{X}(t)) S(t) d W(t), \\
S(0) & =s>0 .
\end{aligned}
$$

Here we allow the flexibility that the appreciation rate and the volatility of the share depend on both the time $t$ and the share price level $S(t)$ for each economic regime.

We now define the discounted price process of the share $S$, which will be used when we discuss the martingale representation result in Section 4.

Let $\xi:=\{\xi(t) \mid t \in \mathcal{T}\}$ be the discounted price process of the share $S$, where $\xi(t):=$ $B^{-1}(t) S(t)=\exp \left(-\int_{0}^{t} r(u) d u\right) S(t)$. Then under $P$, the discounted price process $\xi^{1}$ evolves over time as

$$
\begin{aligned}
d \xi(t) & =(\mu(t, B(t) \xi(t), \mathbf{X}(t))-r(t)) \xi(t) d t+\sigma(t, B(t) \xi(t), \mathbf{X}(t)) \xi(t) d W(t), \\
\xi(0) & =s:=z_{0}>0 .
\end{aligned}
$$

Define $f(\cdot, \cdot, \cdot): \mathcal{T} \times \Re^{+} \times \mathcal{E} \rightarrow \Re$ and $g(\cdot, \cdot, \cdot): \mathcal{T} \times \Re^{+} \times \mathcal{E} \rightarrow \Re^{+}$by

$$
\begin{aligned}
& f(t, \xi(t), \mathbf{X}(t)):=(\mu(t, B(t) \xi(t), \mathbf{X}(t))-r(t)) \xi(t), \\
& g(t, \xi(t), \mathbf{X}(t)):=\sigma(t, B(t) \xi(t), \mathbf{X}(t)) \xi(t) .
\end{aligned}
$$

Under the measurability, differentiability, and boundedness conditions for the functions $\mu$ and $\sigma$ as well as their derivatives, $f\left(t, \xi, \mathbf{e}_{i}\right)$ and $g\left(t, \xi, \mathbf{e}_{i}\right)$ are measurable functions that are three times differentiable in $\xi$, and which, together with their derivatives, are bounded for each $t \in \mathcal{T}$ and $i=1,2, \cdots, N$.

Then under $P$, the discounted price process $\xi$ of the share $S$ can be written as:

$$
\xi(t)=z_{0}+\int_{0}^{t} f(u, \xi(u), \mathbf{X}(u)) d u+\int_{0}^{t} g(u, \xi(u), \mathbf{X}(u)) d W(u) .
$$

Since there are two primitive securities $B$ and $S$ as well as two random factors $W$ and $\mathbf{X}$, the Markov, regime-switching market is incomplete. Consequently, not all contingent claims can be perfectly hedged.

\section{A pricing kernel}

In this section we construct a pricing kernel that incorporates both the financial and economic risks. The pricing kernel is specified by the product of two density processes: one for a measure change for the standard Brownian motion $W$ and the other one for a measure change of the Markov chain. The product of two density processes together with a Girsanov transform for Markov chains were used in Elliott and Siu [17].

Firstly, we define a density process for a measure change for the standard Brownian motion $W$. For each $t \in \mathcal{T}$, let $\mathcal{G}(t):=\mathcal{F}^{\mathbf{X}}(t) \vee \mathcal{F}^{W}(t)$, the minimal $\sigma$-field generated by $\mathcal{F}^{\mathbf{X}}(t)$ and $\mathcal{F}^{W}(t)$. Write $G:=\{\mathcal{G}(t) \mid t \in \mathcal{T}\}$. The enlarged filtration $G$ represents the flow of observable information. 
Suppose $\theta(\cdot, \cdot, \cdot): \mathcal{T} \times \Re^{+} \times \mathcal{E} \rightarrow \Re$ is a measurable function defined by

$$
\theta(t, \xi(t), \mathbf{X}(t)):=\frac{f(t, \xi(t), \mathbf{X}(t))}{g(t, \xi(t), \mathbf{X}(t))},
$$

so $\{\theta(t, \xi(t), \mathbf{X}(t)) \mid t \in \mathcal{T}\}$ is a measurable, $G$-adapted, process. Note that $\theta$ satisfies the same measurability and differentiability conditions as $f$ and $g$. We assume that $\theta$ also satisfies the same boundedness conditions as $f$ and $g$.

We suppose further that for each $i=1,2, \cdots, N$ and $t \in \mathcal{T}, \theta\left(t, \cdot, \mathbf{e}_{i}\right)$ satisfies a linear growth condition in $\xi$ :

$$
\left|\theta\left(t, \xi, \mathbf{e}_{i}\right)\right| \leq K(1+|\xi|) .
$$

Here $K$ is a positive constant, and we suppose that $g(t, \xi, \mathbf{x})>0$ for all $(t, \xi, \mathbf{x}) \in$ $\mathcal{T} \times \Re^{+} \times \mathcal{E}$.

Define a real-valued, $G$-adapted, exponential process $\Lambda^{\theta}:=\left\{\Lambda^{\theta}(t) \mid t \in \mathcal{T}\right\}$ associated with $\theta$ by putting

$$
\Lambda^{\theta}(t):=1+\int_{0}^{t} \theta(u, \xi(u), \mathbf{X}(u)) \Lambda^{\theta}(u) d W(u) .
$$

Note that $\Lambda^{\theta}$ is a $(G, P)$-martingale.

In what follows, we discuss a Girsanov transform for the Markov chain $\mathbf{X}$. The development here follows that of Dufour and Elliott [8]. We only present the key ideas and the main results. For a full account and the proofs of the results, we refer to Dufour and Elliott [8].

Let $\mathbf{C}:=\{\mathbf{C}(t) \mid t \in \mathcal{T}\}$ be an $F^{W}$-predictable, matrix-valued process on $(\Omega, \mathcal{F})$, where $\mathbf{C}(t):=\left[c_{i j}(t)\right]_{i, j=1,2, \cdots, N}$ for each $t \in \mathcal{T}$. We suppose that for each $i, j=$ $1,2, \cdots, N$, the process $\left\{c_{i j}(t) \mid t \in \mathcal{T}\right\}$ satisfies the following conditions, which are also satisfied by the components in an intensity matrix of a Markov chain:

1. $c_{i j}(t) \geq 0$, for $i \neq j$;

2. $\sum_{j=1}^{N} c_{j i}(t)=0$, so $c_{i i}(t) \leq 0$.

We wish to find a new probability measure under which $\mathbf{C}(t):=\left[c_{i j}(t)\right]_{i, j=1,2, \cdots, N}$, $t \in \mathcal{T}$, is a second family of rate matrices of the chain $\mathbf{X}$.

Write, for each $t \in \mathcal{T}$,

$$
\mathbf{D}^{\mathbf{C}}(t):=\left[c_{i j}(t) / a_{i j}(t)\right]_{i, j=1,2, \ldots, N}=:\left[d_{i j}^{\mathbf{C}}(t)\right] .
$$

Note that $a_{i j}(t)>0$ for each $t \in \mathcal{T}$, so $\mathbf{D}^{\mathbf{C}}(t)$ is well-defined.

For each $t \in \mathcal{T}$, let

$$
\mathbf{d}^{\mathbf{C}}(t):=\left(d_{11}^{\mathbf{C}}(t), d_{22}^{\mathbf{C}}(t), \cdots, d_{N N}^{\mathbf{C}}(t)\right)^{\prime} \in \Re^{N} .
$$

Define, for each $t \in \mathcal{T}$,

$$
\mathbf{D}_{0}^{\mathbf{C}}(t):=\mathbf{D}^{\mathbf{C}}(t)-\operatorname{diag}\left(\mathbf{d}^{\mathbf{C}}(t)\right) .
$$

Here $\operatorname{diag}(\mathbf{y})$ is a diagonal matrix with diagonal elements given by the vector $\mathbf{y}$. Similarly, for each $t \in \mathcal{T}, \mathbf{A}_{0}(t)$ is defined as

$$
\mathbf{A}_{0}(t):=\mathbf{A}(t)-\operatorname{diag}(\mathbf{a}(t))
$$


where $\mathbf{a}(t):=\left(a_{11}(t), a_{22}(t), \cdots, a_{N N}(t)\right)^{\prime} \in \Re^{N}$.

Let $\mathbf{N}:=\{\mathbf{N}(t) \mid t \in \mathcal{T}\}$ be a vector-valued counting process defined on $(\Omega, \mathcal{F}, \mathcal{P})$, where for each $t \in \mathcal{T}, \mathbf{N}(t):=\left(N_{1}(t), N_{2}(t), \cdots, N_{N}(t)\right)^{\prime} \in \Re^{N}$ and $N_{j}(t)$ counts the number of jumps of the chain $\mathbf{X}$ to state $\mathbf{e}_{j}$ up to time $t$, for each $j=1,2, \cdots, N$. Then Dufour and Elliott [8] showed that the process $\tilde{\mathbf{N}}:=\{\tilde{\mathbf{N}}(t) \mid t \in \mathcal{T}\}$ defined by

$$
\tilde{\mathbf{N}}(t):=\mathbf{N}(t)-\int_{0}^{t} \mathbf{A}_{0}(u) \mathbf{X}(u-) d u, \quad t \in \mathcal{T},
$$

is an $\Re^{N}$-valued, $\left(F^{\mathbf{X}}, P\right)$-martingale.

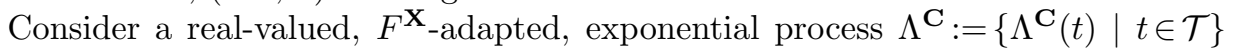
on $(\Omega, \mathcal{F}, P)$ associated with $\mathbf{C}$ defined by setting

$$
\Lambda^{\mathbf{C}}(t)=1+\int_{0}^{t} \Lambda^{\mathbf{C}}(u-)\left[\mathbf{D}_{0}^{\mathbf{C}}(u) \mathbf{X}(u-)-\mathbf{1}\right]^{\prime} d \tilde{\mathbf{N}}(u) .
$$

Here $\mathbf{1}:=(1,1, \ldots, 1)^{\prime} \in \Re^{N}$.

Note that $\Lambda^{\mathbf{C}}$ is an $\left(F^{\mathbf{X}}, P\right)$-(local)-martingale. We suppose that $\mathbf{C}$ is such that $\Lambda^{\mathbf{C}}$ is an $\left(F^{\mathbf{X}}, P\right)$-martingale. Here we use $\Lambda^{\mathbf{C}}$ as the density process for a measure change for the chain $\mathbf{X}$.

Consider a real-valued, $G$-adapted process $\Lambda^{\theta, \mathbf{C}}:=\left\{\Lambda^{\theta, \mathbf{C}}(t) \mid t \in \mathcal{T}\right\}$ on $(\Omega, \mathcal{F}, P)$ defined by the product of the two density processes $\Lambda^{\theta}$ and $\Lambda^{\mathbf{C}}$ :

$$
\Lambda^{\theta, \mathbf{C}}(t):=\Lambda^{\theta}(t) \cdot \Lambda^{\mathbf{C}}(t), \quad t \in \mathcal{T} .
$$

Our assumptions then ensure that $\Lambda^{\theta, \mathbf{C}}$ is a $(G, P)$-martingale.

We now define a probability measure $P^{\theta, \mathbf{C}}$, absolutely continuous with respect to $P$ on $\mathcal{G}(T)$, as

$$
\left.\frac{d P^{\theta, \mathbf{C}}}{d P}\right|_{\mathcal{G}(T)}:=\Lambda^{\theta, \mathbf{C}}(T) .
$$

Then by an extended Girsanov's theorem for the Markov-modulated Brownian motion, the process defined by

$$
W^{\theta}(t):=W(t)-\int_{0}^{t} \theta(u, \xi(u), \mathbf{X}(u)) d u, \quad t \in \mathcal{T},
$$

is a $\left(G, P^{\theta, \mathbf{C}}\right)$-standard Brownian motion.

Consequently under $P^{\theta, \mathbf{C}}$ the discounted share price process $\xi$ follows:

$$
\xi(t)=z_{0}+\int_{0}^{t} g(u, \xi(u), \mathbf{X}(u)) d W^{\theta}(u),
$$

so $\xi$ is a $\left(G, P^{\theta, \mathbf{C}}\right)$-(local)-martingale. We suppose that $\xi$ is a $\left(G, P^{\theta, \mathbf{C}}\right)$-martingale. Further, using a Girsanov transform for the Markov chain, Dufour and Elliott [8] showed that under $P^{\theta, \mathbf{C}}$, the chain $\mathbf{X}$ has a family of rate matrices $\mathbf{C}$, and hence, the following semimartingale dynamics:

$$
\mathbf{X}(t)=\mathbf{X}(0)+\int_{0}^{t} \mathbf{C}(u) \mathbf{X}(u-) d u+\mathbf{M}^{\mathbf{C}}(t) .
$$


Here $\mathbf{M}^{\mathbf{C}}:=\left\{\mathbf{M}^{\mathbf{C}}(t) \mid t \in \mathcal{T}\right\}$ is an $\Re^{N}$-valued, $\left(G, P^{\theta, \mathbf{C}}\right)$-martingale.

To specify a pricing kernel $P^{\theta, \mathbf{C}}$, we must determine both $\theta$ and $\mathbf{C}$. We have already determined the process $\theta$ so that the discounted price process $\xi$ is a $\left(G, P^{\theta, \mathbf{C}}\right)$ (local)-martingale. However, this (local)-martingale condition cannot determine the matrix-valued process $\mathbf{C}$. We shall discuss how to determine $\mathbf{C}$ by minimizing the residual risk of incomplete hedging in Section 5 .

\section{A martingale representation}

We establish a martingale representation result for a general contingent claim. A price of the claim is represented as a sum of two stochastic integrals, one with respect to the standard Brownian motion and another one with respect to the martingale associated with the Markov chain under the measure $P^{\theta, \mathbf{C}}$ defined in the last section. Note that the integrators of the two stochastic integrals are orthogonal to each other under $P^{\theta, \mathbf{C}}$. Then we identify the integrands of the two stochastic integrals explicitly using the concept of stochastic flows.

Firstly, we discuss the concept of stochastic flows. For each $t, s \in \mathcal{T}$ with $t \geq s$ and $z \in \Re^{+}$, let $\xi_{s, t}(z)$ be the unique, strong solution of the stochastic differential equation (2.3) governing the discounted share price under $P$ with initial condition $\xi_{s, s}(z)=z$. Then, under $P$,

$$
\xi_{s, t}(z)=z+\int_{s}^{t} f\left(u, \xi_{s, u}(z), \mathbf{X}(u)\right) d u+\int_{s}^{t} g\left(u, \xi_{s, u}(z), \mathbf{X}(u)\right) d W(u) .
$$

Using similar arguments to those in Kunita [22, 23], Bismut [3] and Elliott and Kopp [13], there is a flow of diffeomorphisms $z \rightarrow \xi_{s, t}(z)$ associated with the solution $\xi_{s, t}(z)$. Write

$$
D_{s, t}(z):=\frac{\partial \xi_{s, t}(z)}{\partial z}
$$

so under $P, D_{s, t}(z)$ satisfies the following linearized equation:

$$
D_{s, t}(z)=1+\int_{s}^{t} f_{\xi}\left(u, \xi_{s, u}(z), \mathbf{X}(u)\right) D_{s, u}(z) d u+\int_{s}^{t} g_{\xi}\left(u, \xi_{s, u}(z), \mathbf{X}(u)\right) D_{s, u}(z) d W(u) .
$$

Here $f_{\xi}$ and $g_{\xi}$ are the first derivatives of $f$ and $g$ with respect to $\xi$, respectively.

It can be shown that under $P$, the inverse $D_{s, t}^{-1}(z)$ of $D_{s, t}(z)$ exists and satisfies the following equation:

$$
\begin{aligned}
D_{s, t}^{-1}(z)= & 1-\int_{s}^{t} f_{\xi}\left(u, \xi_{s, u}(z), \mathbf{X}(u)\right) D_{s, u}^{-1}(z) d u-\int_{s}^{t} g_{\xi}\left(u, \xi_{s, u}(z), \mathbf{X}(u)\right) D_{s, u}^{-1}(z) d W(u) \\
& +\int_{s}^{t}\left(g_{\xi}\left(u, \xi_{s, u}(z), \mathbf{X}(u)\right)\right)^{2} d u .
\end{aligned}
$$

With a slight abuse of notation, let $z:=\xi_{0, t}\left(z_{0}\right)$, for each $t \in \mathcal{T}$. Then by the semi-group property of the solution of the stochastic differential equation (2.3),

$$
\xi_{0, T}\left(z_{0}\right)=\xi_{t, T}\left(\xi_{0, t}\left(z_{0}\right)\right)=\xi_{t, T}(z) .
$$

Differentiating with respect to $z_{0}$ then gives

$$
D_{0, T}\left(z_{0}\right)=D_{t, T}(z) D_{0, t}\left(z_{0}\right)
$$


Applying Itô's differentiation rule to $\Lambda^{\theta, \mathbf{C}}$ and using (3.1) and (3.2) as well as the orthogonality between $W$ and $\mathbf{X}$ under $P$ give:

$$
\begin{aligned}
\Lambda^{\theta, \mathbf{C}}(t)= & 1+\int_{0}^{t} \Lambda^{\theta, \mathbf{C}}(u) \theta(u, \xi(u), \mathbf{X}(u)) d W(u) \\
& +\int_{0}^{t} \Lambda^{\theta, \mathbf{C}}(u-)\left[\mathbf{D}_{0}^{\mathbf{C}}(u) \mathbf{X}(u-)-\mathbf{1}\right]^{\prime} d \tilde{\mathbf{N}}(u) .
\end{aligned}
$$

For each $t, s \in \mathcal{T}$ with $t \geq s$ and $z \in \Re^{+}$, let $\mathcal{K}^{\theta, \mathbf{C}}(s, t, z)$ be the unique, strong solution of the stochastic differential equation (4.1) with initial condition $\mathcal{K}^{\theta, \mathbf{C}}(s, s, z)=1$. Then

$$
\begin{aligned}
\mathcal{K}^{\theta, \mathbf{C}}(s, t, z)= & 1+\int_{s}^{t} \mathcal{K}^{\theta, \mathbf{C}}(s, u, z) \theta\left(u, \xi_{s, u}(z), \mathbf{X}(u)\right) d W(u) \\
& +\int_{s}^{t} \mathcal{K}^{\theta, \mathbf{C}}(s, u-, z)\left[\mathbf{D}_{0}^{\mathbf{C}}(u) \mathbf{X}(u-)-\mathbf{1}\right]^{\prime} d \tilde{\mathbf{N}}(u)
\end{aligned}
$$

Consequently, $\mathcal{K}^{\theta, \mathbf{C}}(s, t, z)$ is a stochastic exponential given by

$$
\mathcal{K}^{\theta, \mathbf{C}}\left(0, t, z_{0}\right)=\mathcal{E}\left(\int_{0}^{\cdot} \theta\left(u, \xi_{0, u}\left(z_{0}\right), \mathbf{X}(u)\right) d W(u)+\int_{0}^{\cdot}\left[\mathbf{D}_{0}^{\mathbf{C}}(u) \mathbf{X}(u-)-\mathbf{1}\right]^{\prime} d \tilde{\mathbf{N}}(u)\right)(t),
$$

so it satisfies:

$$
\mathcal{K}^{\theta, \mathbf{C}}\left(0, T, z_{0}\right)=\mathcal{K}^{\theta, \mathbf{C}}\left(0, t, z_{0}\right) \mathcal{K}^{\theta, \mathbf{C}}(t, T, z) .
$$

Here again $z:=\xi_{0, t}\left(z_{0}\right)$.

Then we have the following lemma.

Lemma 4.1. Let $\theta_{\xi}$ be the derivative of $\theta$ with respect to $\xi$. Write, for each $t \in \mathcal{T}$, $z:=\xi_{0, t}\left(z_{0}\right)$, and

$$
\mathcal{L}^{\theta, \mathbf{C}}(t, T, z):=\int_{t}^{T} \theta_{\xi}\left(u, \xi_{t, u}(z), \mathbf{X}(u)\right) D_{t, u}(z) d W^{\theta}(u)
$$

Then

$$
\frac{\partial \mathcal{K}^{\theta, \mathbf{C}}(t, T, z)}{\partial z}=\mathcal{K}^{\theta, \mathbf{C}}(t, T, z) \mathcal{L}^{\theta, \mathbf{C}}(t, T, z)
$$

Proof. First, using the differentiability of solutions of stochastic differential equations,

$$
\begin{aligned}
\frac{\partial \mathcal{K}^{\theta, \mathbf{C}}(t, T, z)}{\partial z}= & \int_{t}^{T} \frac{\partial \mathcal{K}^{\theta, \mathbf{C}}(t, u, z)}{\partial z} \theta\left(u, \xi_{t, u}(z), \mathbf{X}(u)\right) d W(u) \\
& +\int_{t}^{T} \mathcal{K}^{\theta, \mathbf{C}}(t, u, z) \theta_{\xi}\left(u, \xi_{t, u}(z), \mathbf{X}(u)\right) D_{t, u}(z) d W(u) \\
& +\int_{t}^{T} \frac{\partial \mathcal{K}^{\theta, \mathbf{C}}(t, u-, z)}{\partial z}\left[\mathbf{D}_{0}^{\mathbf{C}}(u) \mathbf{X}(u-)-\mathbf{1}\right]^{\prime} d \widetilde{N}(u)
\end{aligned}
$$


Applying Itô's differentiation rule on $\mathcal{K}^{\theta, \mathbf{C}}(t, T, z) \mathcal{L}^{\theta, \mathbf{C}}(t, T, z)$ gives

$$
\begin{aligned}
& \mathcal{K}^{\theta, \mathbf{C}}(t, T, z) \mathcal{L}^{\theta, \mathbf{C}}(t, T, z) \\
= & \int_{t}^{T} \mathcal{K}^{\theta, \mathbf{C}}(t, T, z) \theta_{\xi}\left(u, \xi_{t, u}(z), \mathbf{X}(u)\right) D_{t, u}(z)\left(d W(u)-\theta\left(u, \xi_{t, u}(z), \mathbf{X}(u)\right) d u\right) \\
& +\int_{t}^{T} \mathcal{L}^{\theta, \mathbf{C}}(t, T, z) \mathcal{K}^{\theta, \mathbf{C}}(t, T, z) \theta\left(u, \xi_{t, u}(z), \mathbf{X}(u)\right) d W(u) \\
& +\int_{t}^{T} \mathcal{L}^{\theta, \mathbf{C}}(t, T, z) \mathcal{K}^{\theta, \mathbf{C}}(t, T, z)\left[\mathbf{D}_{0}^{\mathbf{C}}(u) \mathbf{X}(u-)-\mathbf{1}\right]^{\prime} d \tilde{N}(u) \\
& +\int_{t}^{T} \mathcal{K}^{\theta, \mathbf{C}}(t, T, z) \theta\left(u, \xi_{t, u}(z), \mathbf{X}(u)\right) \theta_{\xi}\left(u, \xi_{t, u}(z), \mathbf{X}(u)\right) D_{t, u}(z) d u \\
= & \int_{t}^{T} \mathcal{K}^{\theta, \mathbf{C}}(t, T, z) \theta_{\xi}\left(u, \xi_{t, u}(z), \mathbf{X}(u)\right) D_{t, u}(z) d W(u) \\
& +\int_{t}^{T} \mathcal{L}^{\theta, \mathbf{C}}(t, T, z) \mathcal{K}^{\theta, \mathbf{C}}(t, T, z) \theta\left(u, \xi_{t, u}(z), \mathbf{X}(u)\right) d W(u) \\
& +\int_{t}^{T} \mathcal{L}^{\theta, \mathbf{C}}(t, T, z) \mathcal{K}^{\theta, \mathbf{C}}(t, T, z)\left[\mathbf{D}_{0}^{\mathbf{C}}(u) \mathbf{X}(u-)-\mathbf{1}\right]^{\prime} d \tilde{N}(u) .
\end{aligned}
$$

By the uniqueness of the solution of the stochastic differential equation, we must have

$$
\frac{\partial \mathcal{K}^{\theta, \mathbf{C}}(t, T, z)}{\partial z}=\mathcal{K}^{\theta, \mathbf{C}}(t, T, z) \mathcal{L}^{\theta, \mathbf{C}}(t, T, z) .
$$

Hence the result follows.

We now consider a function $\psi(\cdot, \cdot): \Re^{+} \times \mathcal{E} \rightarrow \Re^{+}$such that for each $i=1,2, \cdots, N$, $\psi\left(\xi, \mathbf{e}_{i}\right)$ is twice differentiable with respect to $\xi$, and $\psi\left(\xi, \mathbf{e}_{i}\right)$ and $\psi_{\xi}\left(\xi, \mathbf{e}_{i}\right)$ are of at most linear growth in $\xi$, where $\psi_{\xi}$ is the first derivative of $\psi$ with respect to $\xi$. We wish to determine a price, at time $t$, for a general contingent claim whose payoff at expiration $T$ is $\psi(S(T), \mathbf{X}(T))$, where $T>t$. For example, if $\mathbf{X}(T)$ is interpreted as the credit rating of a corporation at time $T$, the payoff of the claim is contingent on both the terminal share price $S(T)$ and corporate credit rating $\mathbf{X}(T)$. It is convenient to work with the discounted claim $\widetilde{\psi}(\xi(T))$ as a function of the discounted share price $\xi(T)$, where

$$
\widetilde{\psi}(\xi(T))=B^{-1}(T) \psi(B(T) \xi(T)) .
$$

It is obvious that $\widetilde{\psi}$ has a linear growth.

Consider the square-integrable, $\left(G, P^{\theta, \mathbf{C}}\right)$-martingale $V:=\{V(t) \mid t \in \mathcal{T}\}$ defined by

$$
V(t):=\mathrm{E}^{\theta, \mathbf{C}}[\widetilde{\psi}(\xi(T), \mathbf{X}(T)) \mid \mathcal{G}(t)] .
$$

Here $\mathrm{E}^{\theta, \mathbf{C}}$ is expectation with respect to $P^{\theta, \mathbf{C}}$ and $V(t)$ is interpreted as the discounted price of the claim to time 0 .

Note that $(\xi, \mathbf{X})$ is jointly Markov with respect to the enlarged filtration $G$. So if $\xi(t)=\xi$ and $\mathbf{X}(t)=\mathbf{x}$,

$$
\begin{aligned}
V(t) & :=\mathrm{E}^{\theta, \mathbf{C}}[\widetilde{\psi}(\xi(T), \mathbf{X}(T)) \mid \mathcal{G}(t)] \\
& =\mathrm{E}^{\theta, \mathbf{C}}[\widetilde{\psi}(\xi(T), \mathbf{X}(T)) \mid \xi(t)=\xi, \mathbf{X}(t)=\mathbf{x}] \\
& =: V(t, \xi, \mathbf{x}) .
\end{aligned}
$$


Write, for each $i=1,2, \cdots, N$,

$$
V_{i}:=V\left(t, \xi(t), \mathbf{e}_{i}\right),
$$

and

$$
\mathbf{V}(t, \xi(t)):=\left(V\left(t, \xi(t), \mathbf{e}_{1}\right), V\left(t, \xi(t), \mathbf{e}_{2}\right), \cdots, V\left(t, \xi(t), \mathbf{e}_{N}\right)\right)^{\prime} \in \Re^{N} .
$$

The following theorem gives a martingale representation result for the discounted price process of the claim $V$.

TheOREM 4.2. Let $\gamma:=\{\gamma(t) \mid t \in \mathcal{T}\}$ and $\boldsymbol{\alpha}:=\{\boldsymbol{\alpha}(t) \mid t \in \mathcal{T}\}$ be two G-predictable processes defined by

$$
\begin{aligned}
\gamma(t):= & \mathrm{E}^{\theta, \mathbf{C}}\left[\int_{t}^{T} \theta_{\xi}\left(u, \xi_{0, u}\left(z_{0}\right), \mathbf{X}(u)\right) D_{0, u}\left(z_{0}\right) d W^{\theta}(u) \cdot \widetilde{\psi}\left(\xi_{0, T}\left(z_{0}\right)\right)\right. \\
& \left.+\widetilde{\psi}_{\xi}\left(\xi_{0, T}\left(z_{0}\right)\right) D_{0, T}\left(z_{0}\right) \mid \mathcal{G}(t)\right] D_{0, t}^{-1}\left(z_{0}\right) \xi_{0, t}\left(z_{0}\right) \sigma\left(t, \xi_{0, t}\left(z_{0}\right), \mathbf{X}(t)\right),
\end{aligned}
$$

and

$$
\boldsymbol{\alpha}(t):=\mathbf{V}(t, \xi(t)),
$$

where $\mathbf{V}(t, \xi(t))$ is given by (4.2) such that

$$
\int_{0}^{T} \mathrm{E}\left[\gamma^{2}(t)\right] d t<\infty
$$

and

$$
\int_{0}^{T} \mathrm{E}\left[\|\alpha(t)\|^{2}\right] d t<\infty
$$

where $\|\cdot\|$ is the norm in $\Re^{2}$.

Then $V$ has the following martingale representation:

$$
V(t)=V(0)+\int_{0}^{t} \gamma(u) d W^{\theta}(t)+\int_{0}^{t}\langle\boldsymbol{\alpha}(u), d \mathbf{M}(u)\rangle .
$$

Here $\int_{0}^{t} \gamma(u) d W^{\theta}(t)$ and $\int_{0}^{t}\langle\boldsymbol{\alpha}(u), d \mathbf{M}(u)\rangle$ are orthogonal to each other under $P^{\theta, \mathbf{C}}$.

Proof. First, note that

$$
\xi_{0, T}\left(z_{0}\right)=\xi_{t, T}(z),
$$

and that

$$
\mathcal{K}^{\theta, \mathbf{C}}\left(0, T, z_{0}\right)=\mathcal{K}^{\theta, \mathbf{C}}\left(0, t, z_{0}\right) \mathcal{K}^{\theta, \mathbf{C}}(t, T, z) .
$$

Then by the Bayes' rule, the Markov property, the $\mathcal{G}(t)$-measurability of $\mathcal{K}^{\theta, \mathbf{C}}\left(0, t, z_{0}\right)$, and the fact that $\mathrm{E}\left[\mathcal{K}^{\theta, \mathbf{C}}(t, T, z) \mid \mathcal{G}(t)\right]=1, P$-a.s.,

$$
\begin{aligned}
V(t) & =\mathrm{E}^{\theta, \mathbf{C}}\left[\widetilde{\psi}\left(\xi_{0, T}\left(z_{0}\right), \mathbf{X}(T)\right) \mid \mathcal{G}(t)\right] \\
& =\frac{\mathrm{E}\left[\mathcal{K}^{\theta, \mathbf{C}}\left(0, T, z_{0}\right) \widetilde{\psi}\left(\xi_{0, T}\left(z_{0}\right), \mathbf{X}(T)\right) \mid \mathcal{G}(t)\right]}{\mathrm{E}\left[\mathcal{K}^{\theta, \mathbf{C}}\left(0, T, z_{0}\right) \mid \mathcal{G}(t)\right]} \\
& =\mathrm{E}\left[\mathcal{K}^{\theta, \mathbf{C}}(t, T, z) \widetilde{\psi}\left(\xi_{t, T}(z), \mathbf{X}(T)\right) \mid \mathbf{X}(t)=\mathbf{x}, \xi_{0, t}\left(z_{0}\right)=z\right] \\
& =: V(t, z, \mathbf{x}), P \text {-a.s. }
\end{aligned}
$$


Recall that under $P^{\theta, \mathbf{C}}$,

$$
\xi_{0, t}\left(z_{0}\right)=z_{0}+\int_{0}^{t} \xi_{0, u}\left(z_{0}\right) \sigma\left(u, B(u) \xi_{0, u}\left(z_{0}\right), \mathbf{X}(u)\right) d W^{\theta}(u) .
$$

To simplify the notation, we write

$$
\sigma(t):=\sigma\left(t, \xi_{0, t}\left(z_{0}\right), \mathbf{X}(t)\right)
$$

Applying Itô's differentiation rule to $V(t, z, \mathbf{x})=V\left(t, \xi_{0, t}\left(z_{0}\right), \mathbf{x}\right)$ gives

$$
\begin{aligned}
& V(t) \\
= & V\left(t, \xi_{0, t}\left(z_{0}\right), \mathbf{x}\right) \\
= & V\left(t, z_{0}, \mathbf{X}(0)\right)+\int_{0}^{t}\left[\frac{\partial V\left(u, \xi_{0, u}\left(z_{0}\right), \mathbf{X}(u)\right)}{\partial u}\right. \\
& \left.+\frac{1}{2} \sigma^{2}(u) \xi_{0, u}^{2}\left(z_{0}\right) \frac{\partial^{2} V}{\partial z^{2}}\left(u, \xi_{0, u}\left(z_{0}\right), \mathbf{X}(u)\right)\right] d u \\
& +\int_{0}^{t} \frac{\partial V\left(u, \xi_{0, u}\left(z_{0}\right), \mathbf{X}(u)\right)}{\partial z} \xi_{0, u}\left(z_{0}\right) \sigma(u) d W^{\theta}(u)+\int_{0}^{t}\left\langle\mathbf{V}\left(u, \xi_{0, u}\left(z_{0}\right)\right), \mathbf{C}(u) \mathbf{X}(u)\right\rangle d u \\
& +\int_{0}^{t}\left\langle\mathbf{V}\left(u, \xi_{0, u}\left(z_{0}\right)\right), d \mathbf{M}^{\mathbf{C}}(u)\right\rangle .
\end{aligned}
$$

Since $V$ is martingale, it must be a special semimartingale. By the unique decomposition of a special semimartingale, (i.e. a special semimartingale can be represented uniquely as the sum of a (local)-martingale and a predictable process of (locally) integrable variation), the sum of the finite variation terms in (4.4) must be indistinguishable from the zero process, i.e.

$$
\frac{\partial V\left(t, \xi_{0, t}\left(z_{0}\right), \mathbf{x}\right)}{\partial t}+\frac{1}{2} \sigma^{2}(t) \xi_{0, t}^{2}\left(z_{0}\right) \frac{\partial^{2} V}{\partial z^{2}}\left(t, \xi_{0, t}\left(z_{0}\right), \mathbf{x}\right)+\left\langle\mathbf{V}\left(t, \xi_{0, t}\left(z_{0}\right)\right), \mathbf{C}(t) \mathbf{x}\right\rangle=0
$$

with $V(T, z, \mathbf{x})=\psi(z, \mathbf{x})$. Further, we must have

$$
\begin{aligned}
\gamma(t) & =\frac{\partial V}{\partial z}\left(t, \xi_{0, t}\left(z_{0}\right), \mathbf{x}\right) \sigma(t) \xi_{0, t}\left(z_{0}\right), \\
\boldsymbol{\alpha}(t) & =\mathbf{V}\left(t, \xi_{0, t}\left(z_{0}\right)\right) .
\end{aligned}
$$


From the differentiability and linear growth conditions of $\theta$,

$$
\begin{aligned}
\frac{\partial V}{\partial z}= & \mathrm{E}\left[\frac{\partial \mathcal{K}^{\theta, \mathbf{C}}(t, T, z)}{\partial z} \widetilde{\psi}\left(\xi_{t, T}(z)\right)+\mathcal{K}^{\theta, \mathbf{C}}(t, T, z) \widetilde{\psi}_{\xi}\left(\xi_{t, T}(z)\right) D_{t, T}(z) \mid \mathcal{G}(t)\right] \\
= & \mathrm{E}\left[\int_{t}^{T} \theta_{\xi}\left(u, \xi_{t, u}(z), \mathbf{X}(u)\right) D_{t, u}(z) d W^{\theta}(u) \cdot \widetilde{\psi}\left(\xi_{t, T}(z)\right)\right. \\
& \left.+\mathcal{K}^{\theta, \mathbf{C}}(t, T, z) \widetilde{\psi}_{\xi}\left(\xi_{t, T}(z)\right) D_{t, T}(z) \mid \mathcal{G}(t)\right] \\
= & \mathrm{E}\left[\mathcal{K}^{\theta, \mathbf{C}}(t, T, z) \int_{t}^{T} \theta_{\xi}\left(u, \xi_{0, u}\left(z_{0}\right), \mathbf{X}(u)\right) D_{0, u}\left(z_{0}\right) d W^{\theta}(u) \cdot \widetilde{\psi}\left(\xi_{t, T}(z)\right)\right. \\
& \left.+\mathcal{K}^{\theta, \mathbf{C}}(t, T, z) \widetilde{\psi}_{\xi}\left(\xi_{0, T}\left(z_{0}\right)\right) D_{0, T}\left(z_{0}\right) \mid \mathcal{G}(t)\right] D_{0, t}^{-1}\left(z_{0}\right) \\
= & \mathrm{E}^{\theta, \mathbf{C}}\left[\int_{t}^{T} \theta_{\xi}\left(u, \xi_{0, u}\left(z_{0}\right), \mathbf{X}(u)\right) D_{0, u}\left(z_{0}\right) d W^{\theta}(u) \cdot \widetilde{\psi}\left(\xi_{t, T}(z)\right)\right. \\
& \left.+\widetilde{\psi}_{\xi}\left(\xi_{0, T}\left(z_{0}\right)\right) D_{0, T}\left(z_{0}\right) \mid \mathcal{G}(t)\right] D_{0, t}^{-1}\left(z_{0}\right) .
\end{aligned}
$$

Hence the result follows.

Corollary 4.3. The time-t discounted prices $V\left(t, \xi_{0, t}\left(z_{0}\right), \mathbf{e}_{i}\right), i=1,2, \cdots, N$, satisfy the following system of coupled partial differential equations:

$$
\begin{aligned}
& \frac{\partial V\left(t, \xi_{0, t}\left(z_{0}\right), \mathbf{e}_{i}\right)}{\partial t}+\frac{1}{2} \sigma^{2}\left(t, \xi_{0, t}\left(z_{0}\right), \mathbf{e}_{i}\right) \xi_{0, t}^{2}\left(z_{0}\right) \frac{\partial^{2} V}{\partial z^{2}}\left(t, \xi_{0, t}\left(z_{0}\right), \mathbf{e}_{i}\right) \\
& +\left\langle\mathbf{V}\left(t, \xi_{0, t}\left(z_{0}\right)\right), \mathbf{C}(t) \mathbf{e}_{i}\right\rangle=0
\end{aligned}
$$

with $V\left(T, z, \mathbf{e}_{i}\right)=\psi\left(z, \mathbf{e}_{i}\right)$.

Proof. This follows directly from the regime-switching partial differential equation for $V\left(t, \xi_{0, t}\left(z_{0}\right), \mathbf{x}\right)$ in the proof of Theorem 4.2.

Note that the system of coupled partial differential equations in Corollary 4.3 depends on the family of intensity matrices $\mathbf{C}:=\{\mathbf{C}(t) \mid t \in \mathcal{T}\}$. We shall determine $\mathbf{C}$ by minimizing the risk of incomplete hedging in the next section, and so fix a price of the claim for each economic state.

\section{Pricing and hedging a general claim}

In this section we apply the martingale representation result in the last section to determine the rate matrix $\mathbf{C}$ (hence fixing a pricing kernel), and a hedging strategy using the money market account, the share, and a zero-coupon bond. The rate matrix $\mathbf{C}$ and the hedging strategy are determined so as to minimize the risk of incomplete hedging measured by the variance of the hedging error. This extends the results in Elliott et al. [15].

Firstly, we introduce a zero-coupon bond $P$ with face value one and maturity at time $T$. Then given $\mathcal{F}^{\mathbf{X}}(t)$, a conditional price of the bond at time $t$, denoted as $P\left(t, T \mid \mathcal{F}^{\mathbf{X}}(t)\right)$, is evaluated as

$$
P^{\mathbf{C}}\left(t, T \mid \mathcal{F}^{\mathbf{X}}(t)\right)=\mathrm{E}^{\theta, \mathbf{C}}\left[\exp \left(-\int_{t}^{T} r(u) d u\right) \mid \mathcal{F}^{\mathbf{X}}(t)\right] .
$$


By the Markov property, given $\mathcal{F}^{\mathbf{X}}(t)=\mathbf{x}$, the conditional price of the bond at time $t$ is then given by

$$
\begin{aligned}
P^{\mathbf{C}}\left(t, T \mid \mathcal{F}^{\mathbf{X}}(t)\right) & =\mathrm{E}^{\theta, \mathbf{C}}\left[\exp \left(-\int_{t}^{T} r(u) d u\right) \mid \mathbf{X}(t)=\mathbf{x}\right] \\
& =: P^{\mathbf{C}}(t, T, \mathbf{x}) .
\end{aligned}
$$

Note that the price of the bond depends on the family of intensity matrices $\mathbf{C}:=$ $\{\mathbf{C}(t) \mid t \in \mathcal{T}\}$. Again to fix a price of the bond, we must determine $\mathbf{C}$.

For each $t \in \mathcal{T}$ and $i=1,2, \cdots, N$, let $\phi_{i}^{\mathbf{C}}(t):=P^{\mathbf{C}}\left(t, T, \mathbf{e}_{i}\right)$. Write $\phi^{\mathbf{C}}(t):=$ $\left(\phi_{1}^{\mathbf{C}}(t), \phi_{2}^{\mathbf{C}}(t), \cdots, \phi_{N}^{\mathbf{C}}(t)\right)^{\prime} \in \Re^{N}$. Define, for each $t \in \mathcal{T}, \boldsymbol{\Pi}^{\mathbf{C}}(t):=\operatorname{diag}(\mathbf{r})-\mathbf{C}^{\prime}(t) \in$ $\Re^{N \otimes N}$, where $\operatorname{diag}(\mathbf{r})$ is the $(N \times N)$-diagonal matrix with diagonal elements given by the vector of interest rates $\mathbf{r}$. Using the same method in Elliott and Kopp [13], it can be shown that the $\Re^{N}$-valued process $\phi^{\mathrm{C}}:=\left\{\phi^{\mathrm{C}}(t) \mid t \in \mathcal{T}\right\}$ satisfies the following matrix-valued, first-order, differential equation:

$$
\frac{d \phi^{\mathbf{C}}(t)}{d t}=\left(\operatorname{diag}(\mathbf{r})-\mathbf{C}^{\prime}(t)\right) \phi^{\mathbf{C}}(t)
$$

with terminal condition

$$
\phi^{\mathbf{C}}(T):=\mathbf{1}=(1,1, \cdots, 1)^{\prime} \in \Re^{N} .
$$

The following theorem was due to Elliott and Kopp [13] and gives a formula for the price of the bond.

THeOREM 5.1. For each $t \in \mathcal{T}$,

$$
P(t, T, \mathbf{X}(t))=\left\langle\phi^{\mathbf{C}}(t), \mathbf{X}(t)\right\rangle,
$$

where $\phi^{\mathrm{C}}(t)$ satisfies the differential equation (5.1) associated with the terminal condition.

For each $t \in \mathcal{T}$, let $\widetilde{P}^{\mathbf{C}}(t, T, \mathbf{X}(t))$ be the discounted bond price at time $t$. Then

$$
\begin{aligned}
\widetilde{P}^{\mathbf{C}}(t, T, \mathbf{X}(t)) & =\mathrm{E}^{\theta, \mathbf{C}}\left[\exp \left(-\int_{0}^{T} r(u) d u\right) \mid \mathcal{F}^{\mathbf{X}}(t)\right] \\
& =\left\langle B^{-1}(t) \phi^{\mathbf{C}}(t), \mathbf{X}(t)\right\rangle .
\end{aligned}
$$

This is an $\left(F^{\mathbf{X}}, P^{\theta, \mathbf{C}}\right)$-martingale.

Applying Itô's differentiation rule on $\widetilde{P}^{\mathbf{C}}(t, T, \mathbf{X}(t))$ gives

$$
\begin{aligned}
& \widetilde{P}^{\mathbf{C}}(t, T, \mathbf{X}(t)) \\
= & P^{\mathbf{C}}(0, T, \mathbf{X}(0))+\int_{0}^{t} B^{-1}(u)\left(\left\langle\frac{d \phi^{\mathbf{C}}(u)}{d u}, \mathbf{X}(u)\right\rangle+\left\langle\phi^{\mathbf{C}}(u), \mathbf{C}(u) \mathbf{X}(u)\right\rangle\right. \\
& \left.-r(u)\left\langle\phi^{\mathbf{C}}(u), \mathbf{X}(u)\right\rangle\right) d u+\int_{0}^{t}\left\langle B^{-1}(u) \phi^{\mathbf{C}}(u), d \mathbf{M}^{\mathbf{C}}(u)\right\rangle .
\end{aligned}
$$

Note that $\left\{\widetilde{P}^{\mathbf{C}}\left(t, T, \mathbf{X}^{2}(t)\right)\right\}$ is an $\left(F^{\mathbf{X}}, P^{\theta, \mathbf{C}}\right)$-martingale, so the finite variation terms, which are not martingales, in the above stochastic integral representation must sum to zero. Consequently,

$$
\widetilde{P}^{\mathbf{C}}(t, T, \mathbf{X}(t))=P^{\mathbf{C}}(0, T, \mathbf{X}(0))+\int_{0}^{t}\left\langle B^{-1}(u) \phi^{\mathbf{C}}(u), d \mathbf{M}^{\mathbf{C}}(u)\right\rangle
$$


We now construct a partially hedged portfolio of the claim consisting of the money market account, the share and the zero-coupon bond, which minimizes the residual risk of incomplete hedging.

Let $\eta:=\{\eta(t) \mid t \in \mathcal{T}\}$ be a $G$-predictable, real-valued process such that

$$
\int_{0}^{T} \mathrm{E}\left[\eta^{2}(t)\right] d t<\infty
$$

Here, for each $t \in \mathcal{T}, \eta(t)$ is the number of units of the discounted zero-coupon bond in the hedged portfolio at time $t$.

Define, for each $t \in \mathcal{T}$,

$$
\widetilde{\gamma}(t):=\frac{\gamma(t)}{\sigma\left(t, \xi_{0, t}\left(z_{0}\right), \mathbf{X}(t)\right) \xi_{0, t}\left(z_{0}\right)} .
$$

Then by Theorem 4.2 and (5.2),

$$
\begin{aligned}
V(t)= & \mathrm{E}^{\theta, \mathbf{C}}\left[\widetilde{\psi}\left(\xi_{0, T}\left(z_{0}\right), \mathbf{X}(T)\right)\right]+\int_{0}^{t} \widetilde{\gamma}(u) d \xi_{0, u}\left(z_{0}\right)+\int_{0}^{t} \eta(u) d \widetilde{P}\left(u, \xi_{0, u}\left(z_{0}\right), \mathbf{X}(u)\right) \\
& +\int_{0}^{t}\left\langle\boldsymbol{\alpha}(u)-\eta(u) B^{-1}(u) \boldsymbol{\phi}^{\mathbf{C}}(u), d \mathbf{M}^{\mathbf{C}}(u)\right\rangle
\end{aligned}
$$

For each $t \in \mathcal{T}$, let

$$
R(t, \eta, \mathbf{C}):=\int_{0}^{t}\left\langle\boldsymbol{\alpha}(u)-\eta(u) B^{-1}(u) \phi^{\mathbf{C}}(u), d \mathbf{M}^{\mathbf{C}}(u)\right\rangle,
$$

where $R(t, \eta, \mathbf{C})$ describes the accumulated hedging error to the claim associated with the hedging policy $(\widetilde{\gamma}, \eta):=\{(\widetilde{\gamma}(t), \eta(t)) \mid t \in \mathcal{T}\}$ up to and including time $t$.

Then, by definition, $R(\eta, \mathbf{C}):=\{R(t, \eta, \mathbf{C}) \mid t \in \mathcal{T}\}$ is an $\left(F^{\mathbf{X}}, P^{\theta, \mathbf{C}}\right)$-martingale. Consequently,

$$
\mathrm{E}^{\theta, \mathbf{C}}[R(T, \eta, \mathbf{C})]=0,
$$

and hence the unconditional variance of $R(T, \eta, \mathbf{C})$ is given by

$$
\operatorname{Var}[R(T, \eta, \mathbf{C})]=\mathrm{E}^{\theta, \mathbf{C}}\left[R^{2}(T, \eta, \mathbf{C})\right] .
$$

This describes the residual risk attributed to incomplete hedging associated with the hedged portfolio $(\widetilde{\gamma}, \eta)$.

Our goal is then to select a trading process $\eta$ so as to minimize the residual risk of incomplete hedging. That is to say, we are going to solve the following minimization problem:

$$
\min _{\eta, \mathbf{C}} \mathrm{E}^{\theta, \mathbf{C}}\left[R^{2}(T, \eta, \mathbf{C})\right]
$$

The following theorem gives a closed-form expression for $\mathrm{E}^{\theta, \mathbf{C}}\left[R^{2}(T, \eta, \mathbf{C})\right]$.

Theorem 5.2. For each $t \in \mathcal{T}$, let

$$
\boldsymbol{\zeta}^{\mathbf{C}}(t):=\boldsymbol{\alpha}(t)-\eta(t) B^{-1}(t) \boldsymbol{\phi}^{\mathbf{C}}(t) \in \Re^{N} .
$$


Then

$$
\begin{aligned}
& \mathrm{E}^{\theta, \mathbf{C}}\left[R^{2}(T, \eta, \mathbf{C})\right] \\
= & \mathrm{E}^{\theta, \mathbf{C}}\left[\int_{0}^{T}\left(\boldsymbol{\zeta}^{\mathbf{C}}\right)^{\prime}(t)\left(\operatorname{diag}(\mathbf{C}(t) \mathbf{X}(t))-\operatorname{diag}(\mathbf{X}(t)) \mathbf{C}^{\prime}(t)-\mathbf{C}(t) \operatorname{diag}(\mathbf{X}(t))\right) \boldsymbol{\zeta}^{\mathbf{C}}(t) d t\right] .
\end{aligned}
$$

Proof. The proof resembles that in Elliott et al. [14], so we only give the key steps. First, note that

$$
\mathbf{X}(t) \otimes \mathbf{X}(t)=\operatorname{diag}(\mathbf{X}(t))
$$

where $\otimes$ is the Kronecker tensor product.

Applying Itô's differentiation rule on $\mathbf{X}(t) \otimes \mathbf{X}(t)$ gives

$$
\begin{aligned}
\mathbf{X}(t) \otimes \mathbf{X}(t)= & \operatorname{diag}(\mathbf{X}(0))+\int_{0}^{t} \operatorname{diag}(\mathbf{X}(u)) \mathbf{C}^{\prime}(u) d u+\int_{0}^{t} \mathbf{C}(u) \operatorname{diag}(\mathbf{X}(u)) d u \\
& +\int_{0}^{t} \mathbf{X}(u-) d\left(\mathbf{M}^{\mathbf{C}}(u)\right)^{\prime}+\int_{0}^{t} d \mathbf{M}^{\mathbf{C}}(u) \mathbf{X}^{\prime}(u-)+\left[\mathbf{M}^{\mathbf{C}}, \mathbf{M}^{\mathbf{C}}\right](t-) \\
& -<\mathbf{M}^{\mathbf{C}}, \mathbf{M}^{\mathbf{C}}>(t)+<\mathbf{M}^{\mathbf{C}}, \mathbf{M}^{\mathbf{C}}>(t) \in \Re^{N} \otimes \Re^{N} .
\end{aligned}
$$

Here $\left[\mathbf{M}^{\mathbf{C}}, \mathbf{M}^{\mathbf{C}}\right](t)=\sum_{0<u \leq t}\left(\Delta \mathbf{M}^{\mathbf{C}}(u)\right)\left(\Delta \mathbf{M}^{\mathbf{C}}(u)\right)^{\prime} \in \Re^{N} \otimes \Re^{N}$ and $<\mathbf{M}^{\mathbf{C}}, \mathbf{M}^{\mathbf{C}}>:=$ $\left.\left\{<\mathbf{M}^{\mathbf{C}}, \mathbf{M}^{\mathbf{C}}\right\rangle(t) \quad \mid t \in \mathcal{T}\right\}$ is the unique predictable process such that $\left\{\left[\mathbf{M}^{\mathbf{C}}, \mathbf{M}^{\mathbf{C}}\right](t)-<\mathbf{M}^{\mathbf{C}}, \mathbf{M}^{\mathbf{C}}>(t) \mid t \in \mathcal{T}\right\}$ is a martingale.

It is not difficult to see that

$$
\operatorname{diag}(\mathbf{X}(t))=\operatorname{diag}(\mathbf{X}(0))+\int_{0}^{t} \operatorname{diag}(\mathbf{C}(u) \mathbf{X}(u)) d u+\operatorname{diag}\left(\mathbf{M}^{\mathbf{C}}(t)\right) \in \Re^{N} \otimes \Re^{N} .
$$

Note that

$$
\mathbf{X}(t) \otimes \mathbf{X}(t)=\operatorname{diag}(\mathbf{X}(t))
$$

and that $\{\mathbf{X}(t) \otimes \mathbf{X}(t) \mid t \in \mathcal{T}\}$ is a special semimartingale. Consequently, using the uniqueness of the decomposition of a special semimartingale into a sum of a predictable process and a martingale and comparing (5.3) and (5.4), we get

$$
\begin{aligned}
& <\mathbf{M}^{\mathbf{C}}, \mathbf{M}^{\mathbf{C}}>(t) \\
= & \int_{0}^{t} \operatorname{diag}(\mathbf{C}(u) \mathbf{X}(u)) d u-\int_{0}^{t} \operatorname{diag}(\mathbf{X}(u)) \mathbf{C}^{\prime}(u) d u-\int_{0}^{t} \mathbf{C}(u) \operatorname{diag}(\mathbf{X}(u)) d u .
\end{aligned}
$$

Then by the isometry,

$$
\begin{aligned}
& \mathrm{E}^{\theta, \mathbf{C}}\left[R^{2}(T, \eta)\right] \\
= & \mathrm{E}^{\theta, \mathbf{C}}\left[\left(\int_{0}^{T}\left\langle\boldsymbol{\zeta}^{\mathbf{C}}(t), d \mathbf{M}^{\mathbf{C}}(t)\right\rangle\right)^{2}\right] \\
= & \mathrm{E}^{\theta, \mathbf{C}}\left[\int_{0}^{T}\left(\boldsymbol{\zeta}^{\mathbf{C}}(t)\right)^{\prime} d<\mathbf{M}^{\mathbf{C}}, \mathbf{M}^{\mathbf{C}}>(t) \boldsymbol{\zeta}^{\mathbf{C}}(t)\right]
\end{aligned}
$$

Hence the result follows. 
The following theorem gives the optimal pricing and hedging strategies $\mathbf{C}^{\dagger}$ and $\eta^{\dagger}$ so as to minimize the residual risk of incomplete hedging.

THEOREM 5.3. A solution of the minimization problem

$$
\min _{\eta, \mathbf{C}} \mathrm{E}^{\eta, \mathbf{C}}\left[R^{2}(T, \eta, \mathbf{C})\right]
$$

is given by $\left\{\left(\eta^{\dagger}(t), \mathbf{C}^{\dagger}(t)\right) \mid t \in \mathcal{T}\right\}$ such that

$$
\eta^{\dagger}(t)=\frac{\left(\boldsymbol{\zeta}^{\mathbf{C}^{\dagger}}\right)^{\prime}(t)\left(\operatorname{diag}\left(\mathbf{C}^{\dagger}(t) \mathbf{X}(t)\right)-\operatorname{diag}(\mathbf{X}(t)) \mathbf{C}^{\dagger^{\prime}}(t)-\mathbf{C}^{\dagger}(t) \operatorname{diag}(\mathbf{X}(t))\right) \boldsymbol{\alpha}(t)}{B^{-1}(t)\left(\boldsymbol{\zeta}^{\mathbf{C}^{\dagger}}\right)^{\prime}(t)\left(\operatorname{diag}\left(\mathbf{C}^{\dagger}(t) \mathbf{X}(t)\right)-\operatorname{diag}(\mathbf{X}(t)) \mathbf{C}^{{ }^{\prime}}(t)-\mathbf{C}^{\dagger}(t) \operatorname{diag}(\mathbf{X}(t))\right) \boldsymbol{\zeta}^{\mathbf{C}^{\dagger}(t)}},
$$

and $\mathbf{C}^{\dagger}(t)$ is a solution of the following problem:

$$
\begin{aligned}
& \min _{\mathbf{C}(t)}\left\{( \boldsymbol { \alpha } ( t ) - \eta ^ { \dagger } ( t ) B ^ { - 1 } ( t ) \boldsymbol { \phi } ^ { \mathbf { C } } ( t ) ) ^ { \prime } \left(\operatorname{diag}(\mathbf{C}(t) \mathbf{X}(t))-\operatorname{diag}(\mathbf{X}(t)) \mathbf{C}^{\prime}(t)\right.\right. \\
& \left.-\mathbf{C}(t) \operatorname{diag}(\mathbf{X}(t)))\left(\boldsymbol{\alpha}(t)-\eta^{\dagger}(t) B^{-1}(t) \boldsymbol{\phi}^{\mathbf{C}}(t)\right)\right\}
\end{aligned}
$$

where $\boldsymbol{\alpha}(t)=\mathbf{V}(t, \xi(t))$ and $\boldsymbol{\phi}^{\mathbf{C}}(t)$ satisfies

$$
\frac{d \phi^{\mathbf{C}}(t)}{d t}=\left(\operatorname{diag}(\mathbf{r})-\mathbf{C}^{\prime}(t)\right) \phi^{\mathbf{C}}(t)
$$

with terminal condition

$$
\boldsymbol{\phi}^{\mathbf{C}}(T):=\mathbf{1}=(1,1, \cdots, 1)^{\prime} \in \Re^{N} .
$$

Proof. The result follows from direct differentiation of the objective function in Theorem 5.2.

\section{Extensions to exotic options}

In this section we apply the martingale representation result in Section 4 and its associate pricing and hedging method in Section 5 to price an Asian option and an American-style option. The techniques used here are similar to those used in Bensoussan and Elliott [2]. However, instead of studying the attainability of the Asian option and the American-style option, we develop here an incomplete hedging strategy based on the money market account, the share and the zero-coupon bond so as to minimize the residual risk of incomplete hedging as in Section 5.

6.1. An Asian option. We consider a general-type of Asian option here with payoff given by the time average for a suitable function $\mathcal{H}\left(\xi_{0, t}\left(z_{0}\right), \mathbf{X}(t)\right)$ of the discounted share price $\xi_{0, t}\left(z_{0}\right)$ and the state of the chain $\mathbf{X}(t)$.

For each $t \in \mathcal{T}$, let

$$
h(t):=\frac{1}{T} \int_{0}^{t} \mathcal{H}\left(\xi_{0, u}\left(z_{0}\right), \mathbf{X}(u)\right) d u .
$$

Then as in Section 4, given $\mathcal{G}(t)$, a conditional discounted price of the Asian option at time $t$ can be evaluated using an equivalent martingale measure $P^{\theta, \mathbf{C}}$ as follows:

$$
\begin{aligned}
& \mathrm{E}^{\theta, \mathbf{C}}\left[\frac{1}{T} \int_{0}^{T} \mathcal{H}\left(\xi_{0, t}\left(z_{0}\right), \mathbf{X}(t)\right) d t \mid \mathcal{G}(t)\right] \\
:= & \frac{1}{T} \int_{0}^{t} \mathcal{H}\left(\xi_{0, t}\left(z_{0}\right), \mathbf{X}(u)\right) d u+\frac{1}{T} \mathrm{E}^{\theta, \mathbf{C}}\left[\int_{t}^{T} \mathcal{H}\left(\xi_{0, u}\left(z_{0}\right), \mathbf{X}(u)\right) d u \mid \xi_{0, t}\left(z_{0}\right)=z, \mathbf{X}(t)=\mathbf{x}\right] \\
= & h(t)+V^{A \operatorname{sian}}(t, z, \mathbf{x}),
\end{aligned}
$$


where

$$
V^{A \operatorname{sian}}(t, z, \mathbf{x}):=\mathrm{E}^{\theta, \mathbf{C}}\left[\frac{1}{T} \int_{t}^{T} \mathcal{H}\left(\xi_{t, u}(z), \mathbf{X}(u)\right) d u \mid \xi_{0, t}\left(z_{0}\right)=z, \mathbf{X}(t)=\mathbf{x}\right] .
$$

Using the same arguments as in Section 4 and Section 5 gives

$$
\begin{aligned}
& \mathrm{E}^{\theta, \mathbf{C}}\left[\frac{1}{T} \int_{0}^{T} \mathcal{H}(\xi(t), \mathbf{X}(t)) d t \mid \mathcal{G}(t)\right] \\
= & \mathrm{E}^{\theta, \mathbf{C}}\left[\frac{1}{T} \int_{0}^{T} \mathcal{H}\left(\xi_{0, t}\left(z_{0}\right), \mathbf{X}(t)\right) d t\right]+\int_{0}^{t} \frac{\partial V^{A s i a n}}{\partial z}(u, z, \mathbf{X}(u)) d \xi_{0, u}\left(z_{0}\right) \\
& +\int_{0}^{t} \eta(u) d \widetilde{P}^{\mathbf{C}}\left(u, \xi_{0, u}\left(z_{0}\right), \mathbf{X}(u)\right)+\int_{0}^{t}\left\langle\boldsymbol{\alpha}(u)-\eta(u) B^{-1}(u) \phi^{\mathbf{C}}(u), d \mathbf{M}^{\mathbf{C}}(u)\right\rangle .
\end{aligned}
$$

To price and hedging the Asian option, we must determine the quantities $\eta(t), \mathbf{C}(t)$ and $\frac{\partial V^{A s i a n}}{\partial z}(t, z, \mathbf{X}(t))$. The quantities $\frac{\partial V^{A s i a n}}{\partial z}(t, z, \mathbf{X}(t))$ and $\eta(t)$ are the numbers of units invested in the (discounted) share and the (discounted) zero-coupon bond, respectively. The matrix $\mathbf{C}$ determines a pricing kernel. Note that both $\eta(t)$ and $\mathbf{C}(t)$ are determined by $\eta^{\dagger}(t)$ and $\mathbf{C}^{\dagger}(t)$, respectively, in Theorem 5.3, with $\boldsymbol{\alpha}(t)$ being replaced by $\boldsymbol{\alpha}^{\text {Asian }}(t):=\mathbf{V}^{A s i a n}(t, z)=$ $\left(V^{A s i a n}\left(t, z, \mathbf{e}_{1}\right), V^{A s i a n}\left(t, z, \mathbf{e}_{2}\right), \cdots, V^{A s i a n}\left(t, z, \mathbf{e}_{N}\right)\right)^{\prime} \in \Re^{N}$. It remains to determine $\frac{\partial V^{A s i a n}}{\partial z}(t, z, \mathbf{X}(t))$; the following theorem gives the result.

TheOREm 6.1. Suppose, for each $i=1,2, \cdots, N, \mathcal{H}\left(\xi, \mathbf{e}_{i}\right)$ is differentiable with respect to $\xi$ and its derivative $\mathcal{H}_{\xi}\left(\xi, \mathbf{e}_{i}\right)$ is integrable. Then for each $t \in \mathcal{T}$,

$$
\begin{aligned}
& \frac{\partial V^{A s i a n}}{\partial z}(t, z, \mathbf{X}(t)) \\
= & \frac{1}{T} \mathrm{E}^{\theta, \mathbf{C}}\left[\int_{t}^{T} \theta_{\xi}\left(u, \xi_{0, u}\left(z_{0}\right), \mathbf{X}(u)\right) D_{0, u}\left(z_{0}\right) d W^{\theta}(u) \cdot \int_{t}^{T} \mathcal{H}\left(\xi_{0, u}\left(z_{0}\right), \mathbf{X}(u)\right) d u\right. \\
& \left.+\int_{t}^{T} \mathcal{H}_{\xi}\left(\xi_{0, u}\left(z_{0}\right), \mathbf{X}(u)\right) D_{0, u}\left(z_{0}\right) d u \mid \mathcal{G}(t)\right] D_{0, t}^{-1}\left(z_{0}\right) .
\end{aligned}
$$

Proof. Using similar arguments as in the proof of Theorem 4.2,

$$
\begin{aligned}
& V^{A \operatorname{sian}}(t, z, \mathbf{X}(t)) \\
= & \mathrm{E}^{\theta, \mathbf{C}}\left[\frac{1}{T} \int_{t}^{T} \mathcal{H}\left(\xi_{t, u}(z), \mathbf{X}(u)\right) d u \mid \mathcal{G}(t)\right] \\
= & \frac{\mathrm{E}\left[\mathcal{K}^{\theta, \mathbf{C}}\left(0, T, z_{0}\right) \frac{1}{T} \int_{t}^{T} \mathcal{H}\left(\xi_{t, u}(z), \mathbf{X}(u)\right) d u \mid \mathcal{G}(t)\right]}{\mathrm{E}\left[\mathcal{K}^{\theta, \mathbf{C}}\left(0, T, z_{0}\right) \mid \mathcal{G}(t)\right]} \\
= & \frac{1}{T} \mathrm{E}\left[\mathcal{K}^{\theta, \mathbf{C}}(t, T, z) \int_{t}^{T} \mathcal{H}\left(\xi_{t, u}(z), \mathbf{X}(u)\right) d u \mid \mathbf{X}(t)=\mathbf{x}, \xi_{0, t}\left(z_{0}\right)=z\right] .
\end{aligned}
$$

Then the result follows from direct differentiation and Lemma 4.1.

6.2. An American option. We now consider the pricing and hedging of a general American-style contingent claim which gives a discounted reward of $\widehat{\mathcal{H}}_{1}\left(\xi_{0, t}\left(z_{0}\right), \mathbf{X}(t)\right):=B^{-1}(t) \mathcal{H}_{1}\left(\xi_{0, t}\left(z_{0}\right), \mathbf{X}(t)\right)$ at time $t$ when the claim is exercised at time $t$. 
Let $\mathcal{T}\left(t_{1}, t_{2}\right)$ be the set of $G$-stopping times taking values in $\left[t_{1}, t_{2}\right]$. Then given $\mathcal{G}(t)$, a conditional price of the American-style claim at time $t$ is given by

$$
\begin{aligned}
& \operatorname{ess} \sup _{\tau \in \mathcal{T}(t, T)} \mathrm{E}^{\theta, \mathbf{C}}\left[B(t) \widehat{\mathcal{H}}_{1}\left(\xi_{0, \tau}\left(z_{0}\right), \mathbf{X}(\tau)\right) \mid \mathcal{G}(t)\right] \\
= & \underset{\tau \in \mathcal{T}(t, T)}{\operatorname{ess} \sup } \mathrm{E}^{\theta, \mathbf{C}}\left[B(t) \widehat{\mathcal{H}}_{1}\left(\xi_{t, \tau}(z), \mathbf{X}(\tau)\right) \mid \mathbf{X}(t)=\mathbf{x}, \xi_{0, t}\left(z_{0}\right)=z\right] \\
= & : V^{\text {American }}(t, z, \mathbf{x}) .
\end{aligned}
$$

Under certain conditions (see Bensoussan and Lions [1] or Krylov [21]), the solution to the above optimal stopping problem $V^{\text {American }}(t, z, \mathbf{x})$ is such that for each $i=1,2, \cdots, N, V^{\text {American }}\left(t, z, \mathbf{e}_{i}\right)$ is in the Sobolev space $W^{1,2}$. Consequently, a generalized Itô's differentiation rule can be applied to $V^{\text {American }}(t, z, \mathbf{x})$.

Suppose $\tau^{\dagger}(t)$ is the smallest optimal stopping time in $\mathcal{T}(t, T)$. Then

$$
V^{A m e r i c a n}(t, z, \mathbf{x})=\mathrm{E}^{\theta, \mathbf{C}}\left[B(t) \widehat{\mathcal{H}}_{1}\left(\xi_{t, \tau^{\dagger}(t)}(z), \mathbf{X}\left(\tau^{\dagger}\right)\right) \mid \mathbf{X}(t)=\mathbf{x}, \xi_{0, t}\left(z_{0}\right)=z\right] .
$$

Consequently, the Snell envelope is given by

$$
\begin{aligned}
& J(t):=\operatorname{ess}_{\tau \in \mathcal{T}(t, T)} \mathrm{sup}^{\theta, \mathbf{C}}\left[\widehat{\mathcal{H}}_{1}\left(\xi_{t, \tau}(z), \mathbf{X}(\tau)\right) \mid \mathcal{G}(t)\right] \\
& =B^{-1}(t) V^{\text {American }}(t, z, \mathbf{x}) \\
& =\mathrm{E}^{\theta, \mathbf{C}}\left[\widehat{\mathcal{H}}_{1}\left(\xi_{t, \tau^{\dagger}(t)}(z), \mathbf{X}\left(\tau^{\dagger}\right)\right) \mid \mathbf{X}(t)=\mathbf{x}, \xi_{0, t}\left(z_{0}\right)=z\right] .
\end{aligned}
$$

That is, $\{J(t) \mid t \in \mathcal{T}\}$ is the smallest $\left(G, P^{\theta, \mathbf{C}}\right)$-supermartingale which majorizes the discounted reward. For some suitable function $\mathcal{H}_{1},\{J(t) \mid t \in \mathcal{T}\}$ is of class $D$, so it has a Doob-Meyer decomposition

$$
J(t)=\widehat{M}(t)-\Lambda(t)
$$

Here $\{\widehat{M}(t) \mid t \in \mathcal{T}\}$ is a $\left(G, P^{\theta, \mathbf{C}}\right)$-martingale and $\{\Lambda(t) \mid t \in \mathcal{T}\}$ is a unique, predictable, non-decreasing process with $\Lambda(0)=0$.

In what follows, we derive a pricing and hedging strategy for the American-style claim in the common continuation region, say $\mathcal{C}:=\mathcal{C}_{1} \cup \mathcal{C}_{2} \cup \cdots \cup \mathcal{C}_{N}$, where, for each $i=1,2, \cdots, N$,

$$
\mathcal{C}_{i}:=\left\{(t, z) \in \mathcal{T} \times \Re^{+} \mid V^{\text {American }}\left(t, z, \mathbf{e}_{i}\right)>\mathcal{H}_{1}\left(z, \mathbf{e}_{i}\right)\right\} .
$$

In the common continuation region $\mathcal{C}, d \Lambda(t)=0$ and $\tau^{\dagger}(t)>t$. Consequently,

$$
\begin{aligned}
J(t) & =\mathrm{E}^{\theta, \mathbf{C}}\left[\widehat{\mathcal{H}}_{1}\left(\xi_{t, \tau^{\dagger}(t)}(z), \mathbf{X}\left(\tau^{\dagger}\right)\right) \mid \mathbf{X}(t)=\mathbf{x}, \xi_{0, t}\left(z_{0}\right)=z\right] \\
& =B^{-1}(t) V^{A \text { merican }}(t, z, \mathbf{x})=\widehat{M}(t), \quad t \in \mathcal{T},
\end{aligned}
$$

which is a $\left(G, P^{\theta, \mathbf{C}}\right)$-martingale.

Again, as in Section 4 and Section $5,\{J(t) \mid t \in \mathcal{T}\}$ has the following martingale representation:

$$
\begin{aligned}
J(t)= & \mathrm{E}^{\theta, \mathbf{C}}\left[\widehat{\mathcal{H}}_{1}\left(\xi_{0, \tau^{\dagger}(t)}\left(z_{0}\right), \mathbf{X}\left(\tau^{\dagger}\right)\right)\right]+\int_{0}^{t} \frac{\partial V^{\text {American }}}{\partial z}\left(u, \xi_{0, u}\left(z_{0}\right), \mathbf{X}(u)\right) d \xi_{0, u}\left(z_{0}\right) \\
& +\int_{0}^{t} \eta(u) d \widetilde{P}^{\mathbf{C}}\left(u, \xi_{0, u}\left(z_{0}\right), \mathbf{X}(u)\right)+\int_{0}^{t}\left\langle\boldsymbol{\alpha}(u)-\eta(u) B^{-1}(u) \boldsymbol{\phi}^{\mathbf{C}}(u), d \mathbf{M}^{\mathbf{C}}(u)\right\rangle .
\end{aligned}
$$


Consequently, with $B(t)=\exp \left(\int_{0}^{t} r(u) d u\right)$,

$$
\begin{aligned}
& V^{A m e r i c a n}(t, z, \mathbf{X}) \\
= & \mathrm{E}^{\theta, \mathbf{C}}\left[\widehat{\mathcal{H}}_{1}\left(\xi_{0, \tau^{\dagger}(t)}\left(z_{0}\right), \mathbf{X}\left(\tau^{\dagger}\right)\right)\right]+\int_{0}^{t} r(u) V^{A m e r i c a n}\left(u, \xi_{0, u}\left(z_{0}\right), \mathbf{X}(u)\right) d u \\
& +\int_{0}^{t} B(u) \frac{\partial V^{A m e r i c a n}}{\partial z}\left(u, \xi_{0, u}\left(z_{0}\right), \mathbf{X}(u)\right) d \xi_{0, u}\left(z_{0}\right) \\
& +\int_{0}^{t} \eta(u) B(u) d \widetilde{P}^{\mathbf{C}}\left(u, \xi_{0, u}\left(z_{0}\right), \mathbf{X}(u)\right) \\
& +\int_{0}^{t}\left\langle B(u) \boldsymbol{\alpha}(u)-\eta(u) \phi^{\mathbf{C}}(u), d \mathbf{M}^{\mathbf{C}}(u)\right\rangle \\
= & \mathrm{E}^{\theta, \mathbf{C}}\left[\widehat{\mathcal{H}}_{1}\left(\xi_{0, \tau^{\dagger}}(t)\left(z_{0}\right), \mathbf{X}\left(\tau^{\dagger}\right)\right)\right]+\int_{0}^{t} r(u)\left(V^{A m e r i c a n}\left(u, \xi_{0, u}\left(z_{0}\right), \mathbf{X}(u)\right)\right. \\
& \left.-\frac{\partial V^{A m e r i c a n}}{\partial z}\left(u, \xi_{0, u}\left(z_{0}\right), \mathbf{X}(u)\right) S(u)-\eta(u) P^{\mathbf{C}}\left(u, \xi_{0, u}\left(z_{0}\right), \mathbf{X}(u)\right)\right) d u \\
& +\int_{0}^{t} \frac{\partial V^{A m e r i c a n}}{\partial z}\left(u, \xi_{0, u}\left(z_{0}\right), \mathbf{X}(u)\right) d S(u)+\int_{0}^{t} \eta(u) d P^{\mathbf{C}}\left(u, \xi_{0, u}\left(z_{0}\right), \mathbf{X}(u)\right) \\
& +\int_{0}^{t}\left\langle B(u) \boldsymbol{\alpha}(u)-\eta(u) \phi^{\mathbf{C}}(u), d \mathbf{M}^{\mathbf{C}}(u)\right\rangle .
\end{aligned}
$$

Consequently, to hedge the American-style claim, we invest $V^{\text {American }}\left(t, \xi_{0, t}\left(z_{0}\right), \mathbf{X}(t)\right)-\frac{\partial V^{\text {American }}}{\partial z}\left(t, \xi_{0, t}\left(z_{0}\right), \mathbf{X}(t)\right) S(t)-\eta(t) P^{\mathbf{C}}\left(t, \xi_{0, t}\left(z_{0}\right), \mathbf{X}(t)\right)$, $\frac{\partial V^{A m e r i c a n}}{\partial z}\left(t, \xi_{0, t}\left(z_{0}\right), \mathbf{X}(t)\right)$ and $\eta(t)$ in the money market account, the share, and the zero-coupon bond at time $t$, respectively. The initial price of the American-style claim is given by $\mathrm{E}^{\theta, \mathbf{C}}\left[\widehat{\mathcal{H}}_{1}\left(\xi_{0, \tau^{\dagger}(t)}\left(z_{0}\right), \mathbf{X}\left(\tau^{\dagger}\right)\right)\right]$ and its early exercise strategy is determined as

$$
\tau^{\dagger}(t):=\inf \left\{\tau \in \mathcal{T}(t, T) \mid V^{\text {American }}(t, z, \mathbf{x}) \leq \mathcal{H}_{1}(z, \mathbf{x})\right\} .
$$

So to determine the price, the early exercise strategy, and the hedging strategy of the American-style claim so as to minimize the residual risk of incomplete hedging, we must determine $\frac{\partial V^{\text {American }}}{\partial z}\left(t, \xi_{0, t}\left(z_{0}\right), \mathbf{X}(t)\right), \eta(t)$ and $\mathbf{C}(t)$. As in Section $4, \eta(t)$, and $\mathbf{C}(t)$ are determined by $\eta^{\dagger}(t)$ and $\mathbf{C}^{\dagger}(t)$ in Theorem 5.3, respectively. The following theorem then determines $\frac{\partial V^{\text {American }}}{\partial z}\left(t, \xi_{0, t}\left(z_{0}\right), \mathbf{X}(t)\right)$.

Theorem 6.2. Suppose, for each $i=1,2, \cdots, N, \mathcal{H}_{1}\left(\xi, \mathbf{e}_{i}\right)$ is differentiable with respect to $\xi$ and its derivative $\frac{\partial \mathcal{H}_{1}\left(\xi, \mathbf{e}_{i}\right)}{\partial \xi}$ is integrable. Then for each $t \in \mathcal{T}$,

$$
\begin{aligned}
& \frac{\partial V^{\text {American }}}{\partial z}(t, z, \mathbf{X}(t)) \\
= & \mathrm{E}^{\theta, \mathbf{C}}\left[\int_{t}^{\tau^{\dagger}(t)} \theta_{\xi}\left(u, \xi_{0, u}\left(z_{0}\right), \mathbf{X}(u)\right) D_{0, u}\left(z_{0}\right) d W^{\theta}(u) \cdot \mathcal{H}_{1}\left(\xi_{0, \tau^{\dagger}(t)}\left(z_{0}\right), \mathbf{X}\left(\tau^{\dagger}(t)\right)\right)\right. \\
& \left.+\frac{\partial \mathcal{H}_{1}\left(\xi_{0, \tau^{\dagger}(t)}\left(z_{0}\right), \mathbf{X}\left(\tau^{\dagger}(t)\right)\right)}{\partial \xi} D_{0, \tau^{\dagger}(t)}\left(z_{0}\right) \mid \mathcal{G}(t)\right] D_{0, t}^{-1}\left(z_{0}\right) .
\end{aligned}
$$

The proof of Theorem 6.2 resembles that of Theorem 6.1. So we only state the result without giving the proof. 


\section{Conclusion}

We discussed the pricing and hedging of general contingent claims in a Markov regime-switching market, where a money market account, a share and a zero-coupon were traded. The payoffs of the general claims depend on both the prices of the share and the states of a Markov chain describing the states of an economy. We introduced a general pricing kernel based on the product of two density processes for measure changes so as to price both the financial and economic risks explicitly. A general martingale representation result was established and its integrands were identified with respect to the general pricing kernel using the concept of stochastic flows. A price and a hedging strategy, which take into account both the financial and economic risks, were then determined by minimizing the variance of the hedging error of a trading portfolio consisting of the money market account, the share and the zero-coupon bond. We also extended the results to price and hedge Asian-style and American-style general contingent claims.

Acknowledgement. We would like to thank ARC for their support.

\section{REFERENCES}

[1] A. Bensoussan and J.L. Lions, Applications of Variational Inequalities in Stochastic Control, New York, North-Holland, 1982.

[2] A. Bensoussan and R.J. Elliott, Attainable claims in a Markov market, Mathematical Finance, 5(2), 121-131, 1995.

[3] J.M. Bismut, Martingales, the Malliavin calculus and hypoellipticity under general Hörmander's conditions, Zeitschrift für Wahrscheinlichkeitstheorie, 56, 469-505, 1981.

[4] P.P. Boyle and T. Draviam, Pricing exotic options under regime switching, Insurance: Mathematics and Economics, 40(2), 267-282, 2007.

[5] J. Buffington and R.J. Elliott, American options with regime switching, International Journal of Theoretical and Applied Finance, 5, 497-514, 2002.

[6] B.D. Colwell, R.J. Elliott and P.E. Kopp, Martingale representation and hedging policies, Stochastic Process and its Applications, 38, 335-345, 1991.

[7] B.D. Colwell and R.J. Elliott, Discontinuous asset prices and nonattainable contingent claims, Mathematical Finance, 3, 295-308, 1993.

[8] F. Dufour and R.J. Elliott, Filtering with discrete state observations, Applied Mathematics and Optimization, 40, 259-272, 1999.

[9] R.J. Elliott, Stochastic Calculus and Applications, Springer-Verlag: Berlin-Heidelberg-New York, 1982.

[10] R.J. Elliott, L. Aggoun and J.B. Moore, Hidden Markov Models: Estimation and Control, Springer-Verlag: Berlin-Heidelberg-New York, 1994.

[11] R.J. Elliott and H. Föllmer, Orthogonal martingale representation. Stochastic Analysis, in honor of Moshe Zakai, eds. E. Mayer-Wolf, E. Merzbach, A. Schwartz, Academic Press, New York, 1991.

[12] R.J. Elliott and P.E. Kopp, Mathematics of Financial Markets, second ed., Springer-Verlag: Berlin-Heidelberg-New York, 2005.

[13] R.J. Elliott, L.L. Chan and T.K. Siu, Option pricing and Esscher transform under regime switching, Annals of Finance, 1(4), 423-432, 2005.

[14] R.J. Elliott, T.K. Siu and H. Yang, Martingale representation for contingent claims with regime switching, Communications on Stochastic Analysis, 1(2), 279-292, 2007.

[15] R.J. Elliott, T.K. Siu, L.L Chan and J.W. Lau, Pricing options under a generalized Markov modulated jump diffusion model, Stochastic Analysis and Applications, 25(4), 821-843, 2007.

[16] R.J. Elliott and T.K. Siu and A. Badescu, On pricing and hedging options in regime-switching models with feedback effect, Haskayne School of Business, University of Calgary, School of Mathematical Sciences, Univesity of Adelaide, Faculty of Business and Economics, Macquarie University, preprint, 2010.

[17] R.J. Elliott and T.K. Siu, On risk minimizing portfolios under a Markovian regime-switching Black-Scholes economy, Annals of Operations Research, 176(1), 271-291, 2010. 
[18] S.M. Goldfeld and Q.E. Quandt, A Markov model for switching regressions, Journal of Econometrics, 1, 3-16, 1973.

[19] X. Guo, Information and option pricings, Quantitative Finance, 1, 38-44, 2001.

[20] J.D. Hamilton, A new approach to the economic analysis of nonstationary time series and the business cycle, Econometrica, 57, 357-384, 1989.

[21] N.V. Krylov, Controlled Diffusion Processes, Springer-Verlag, Berlin-Heidelberg-New York, 1980.

[22] H. Kunita, Stochastic Partial Differential Equations Connected with Nonlinear Filtering, Lecture Notes in Mathematics 972, Springer-Verlag: Berlin-Heidelberg-New York, 1978.

[23] H. Kunita, Stochastic Differential Equations and Stochastic Flows of Diffeomorphisms, Lecture Notes in Mathematics 1097, Springer-Verlag, Berlin-Heidelberg-New York, 1982.

[24] V. Naik, Option valuation and hedging strategies with jumps in the volatility of asset returns, Journal of Finance, 48, 1969-1984, 1993.

[25] S. Pliska, Introduction to Mathematical Finance: Discrete Time Models, Blackwell Publishers, United States, 1997.

[26] R.E. Quandt, The estimation of parameters of linear regression system obeying two separate regimes, Journal of the American Statistical Association, 55, 873-880, 1958.

[27] T.K. Siu, A game theoretic approach to option valuation under Markovian regime-switching models, Insurance: Mathematics and Economics, 42(3), 1146-1158, 2008.

[28] H. Tong, Discussion of a paper by A.J. Lawrance and N.T. Kottegoda, Journal of Royal Statistical Society: Series A, 140, 34-35, 1977.

[29] H. Tong, On a threshold model, C.H. Chen (Eds.), Pattern Recognition and Signal Processing, Sijthoff and Noordhoff, The Netherlands, 575-586, 1978.

[30] H. Tong, A view on non-linear time series model building, O.D. Anderson (Eds.), Time Series, North-Holland, Amsterdam, 41-56, 1980. 